\title{
Intercellular cell adhesion molecule-1 and vascular cell adhesion molecule-1 in children. Do they play a role in the progression of atherosclerosis?
}

\author{
Lampros Fotis, ${ }^{1}$ Dionysios Giannakopoulos, ${ }^{2}$ Lela Stamogiannou, ${ }^{1}$ Maria Xatzipsalti ${ }^{1}$
}

${ }^{1}$ First Department of Pediatrics, P. \& A. Kyriakou Children's Hospital, ${ }^{2}$ Private Practitioner, Athens, Greece

\section{INTRODUCTION}

In 1995 David Barker formulated the 'Barker hypothesis' according to which there is a correlation between undernutrition in utero and several diseases in adult life. More specifically, undernutrition in utero permanently affects the vascular bed, resulting in a predisposition to diseases later on, including hypertension, hypercholesterolemia, stroke, cardiovascular disease and diabetes. ${ }^{1}$ The same can also apply to macrosomic infants who appear to be at an increased risk for obesity and glucose metabolism disorders. ${ }^{2}$ Furthermore, growth during early childhood affects health during adulthood and the prognosis is even worse if this weight gain is combined with a history of small weight for gestational age at birth followed by abrupt weight gain in early childhood. ${ }^{3}$ The fact that chronic deteriorating diseases that develop in adults have their origin in early childhood renders

Key words: Atherosclerosis, Cell adhesion molecules, Childhood obesity, Diabetes mellitus, Hypercholesterolemia, Intercellular adhesion molecule-1, Type 1, Vascular cell adhesion molecule-1

Address for correspondence:

Xatzipsalti Maria, 1 Zafeiriou Str., 17122 Nea Smyrni, Athens, Greece, Tel.: + 30 2104122712, +30 6974456750, Fax: +302132009826, E-mail: marax5873@hotmail.co.uk Received 29-06-11, Revised 12-09-11, Accepted 28-10-11 imperative the discovery of possible interventions that would stop the progression of those diseases during childhood.

Atherosclerosis is one of the chronic diseases of adulthood, with increased morbidity and mortality in both men and women of all racial groups in most westernized societies. Lesions grow slowly during childhood as fatty streaks and form raised lesions in adolescents and young adults. ${ }^{4}$ The mechanisms of atherosclerosis development are complex and include vascular endothelium injury, activation of inflammatory cells (monocytes -macrophages, T-lymphocytes etc) and their migration to the site of the lesion, unverifiable proliferation of smooth muscle cells and the accumulation of fat within the vessel wall. ${ }^{5}$ The most crucial factors involved in endothelial injury are oxidized cholesterol, cytokines, mechanical injury, reactive species and the toxic contents of cigarette smoke and several environmental pollutants. ${ }^{6}$

Endothelium reacts to atherogenic stimuli by expressing on its surface two classes of adhesion molecules, the selectins and the molecules of the immunoglobulin superfamily, mainly intra- or intercellular cell adhesion molecule-1 (ICAM-1) and vascular cell adhesion molecule-1 (VCAM-1). Following this, leucocyte integrins and L-selectins are expressed, causing attraction and adhesion of monocytes and T-lymphocytes on the endothelium, according to the 4-step model (Figure 1). ${ }^{5}$

In the following review we will examine the ad- 


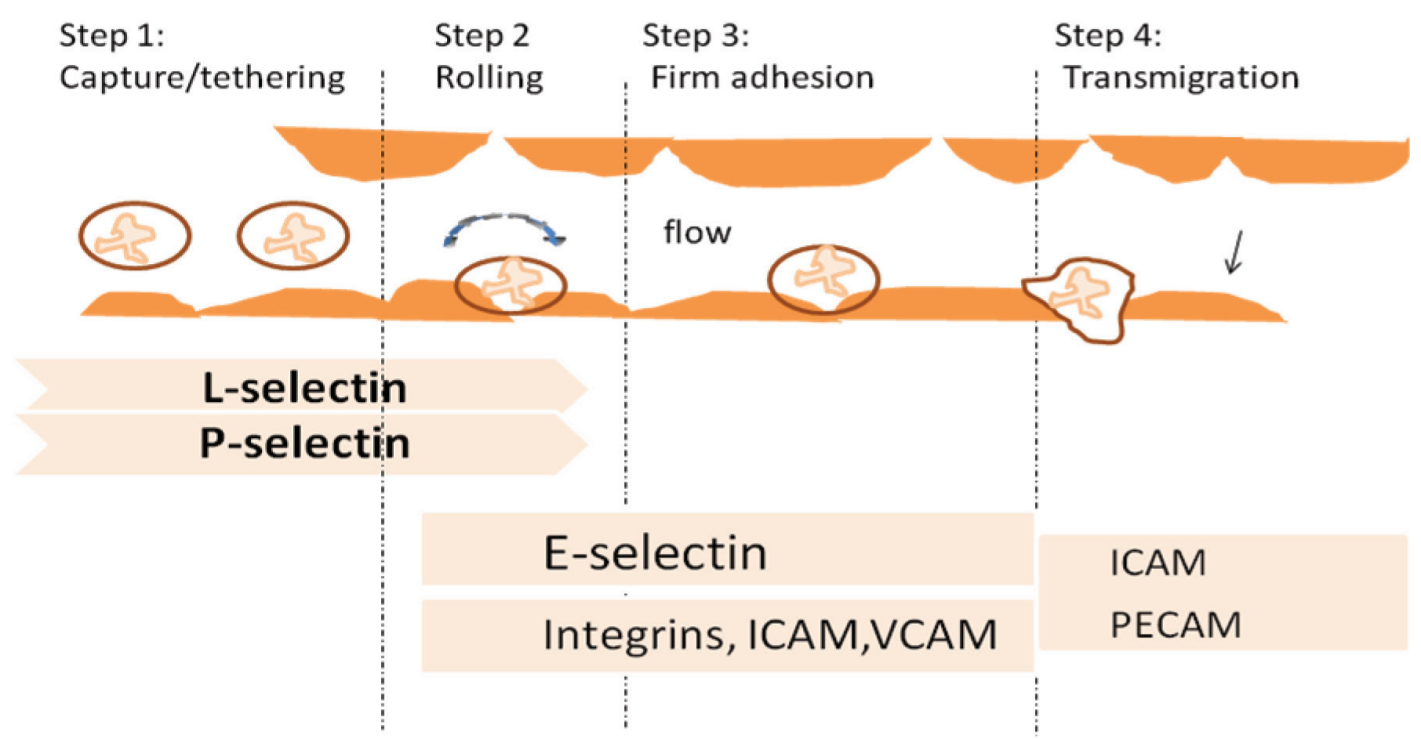

Figure 1. The four-step model of leukocyte adhesion and transmigration across an endothelial monolayer. Leukocyte tethering and rolling (steps 1 and 2) are mediated primarily by selectin-carbohydrate interactions. Firm adhesion (step 3) follows if leukocytes encounter activating signals while rolling along the endothelium. Activation-dependent attachment of b2 integrins (Mac-1, LFA-1) on neutrophils to endothelial ICAM-1 supports this firm or secondary cell adhesion to the vessel wall. Transmigration (step 4) may occur if a favorable chemotactic gradient exists across the monolayer. Platelet/endothelial cell adhesion molecule 1 (PECAM-1) expressed at endothelial cell junctions appears to be required for transmigration by binding homophilically to PECAM-1 expressed on leukocytes.

hesion molecules ICAM-1 and VCAM-1 and their role in both healthy children and subjects prone to atherosclerosis.

\section{ICAM-1}

ICAM-1 is a transmembrane glucoprotein that belongs to the immunoglobulin superfamily. It consists of 5 extracellular immunoglobulin particles, a transmembrane part and a small intracellular part consisting of 28 aminoacids with a $\mathrm{COOH}$ ending. ICAM-1 is expressed predominantly by epithelial and endothelial cells, macrophages, monocytes, B and T-lymphocytes, fibroblasts and dendritic cells of the thymus, the lymph nodes, the gastrointestinal tract, skin, liver and hemopoietic cells of the medulla. ${ }^{7}$ Its main ligands are integrins LFA-1 (CD11a/CD18) and Mac-1 (CD11b/CD18) and it is also a receptor of fibrinogen and hyaluronate, a component of the extracellular matrix. ICAM-1 is crucial in endothelialleucocytes interaction, leucocyte migration at the inflammation site, cellular interaction during antigen presentation where it acts as a cofactor of antigenpresenting cell stimulation, T-lymphocyte activation and bacterial pathogenesis. ${ }^{8}$ Its role is also crucial during extracellular signal transportation leading to changes through the protein kinase $\mathrm{C}$ pathway, cAMP, phospholipase A2, $\mathrm{Ca}+2$ and proteosomes. ${ }^{9}$ ICAM1 levels appear increased in topical or generalized inflammation states such as atherosclerosis, allergic asthma, septic shock, inflammatory and allergic skin diseases, infarction, autoimmune diseases and malignancies. Soluble ICAM-1 (sICAM-1) is produced either by proteolytic cleavage from the cell membrane or directly by cells. It is present in body fluids like cerebrospinal fluid, synovial fluid, saliva, urine and bronchial secretions. ${ }^{10}$

\section{VCAM-1}

VCAM-1 is also a transmembrane glucoprotein member of the immunoglobulin superfamily. Its extracellular part consists of 6 or 7 immunoglobulin particles. ${ }^{11}$ It is expressed by vascular endothelial cells under the influence of inflammatory stimuli as well as in the endothelium of the medulla. ${ }^{12}$ Other cell types that produce VCAM-1 are fibroblasts covering synovial surfaces, chondrocytes, tissue macrophages, 
epithelial cells of the thymus, dendritic cells and pericytes of neural tissues. The main ligand of VCAM-1 is the integrin a4 $\beta 1$ (VLA-4). Integrins a9b1, aDb2, erythrocytes infected by plasmodium falciparum and viruses are also known VCAM-1 ligands. ${ }^{13}$ VCAM-1 additionally interacts with SPARC (secreted protein acidic and rich in cysteine), a molecule that induces cytoskeleton modification and the creation of gaps between cells important for leucocyte transmigration. ${ }^{14}$ VCAM-1 and VLA-4 interaction is important for adhesion of circulating cells to the vascular endothelium, the adhesion of primitive hemopoietic cells to the stromal cells of the medulla, leucocyte-dendritic cell interaction, neurogenesis and myogenesis. ${ }^{15}$ Increased VCAM-1 expression is present in atherosclerotic lesions. Soluble VCAM-1(sVCAM-1) has been detected only in serum and its formation is correlated to the proteolytic cleavage of the molecule at its entry point in the cell membrane. ${ }^{13,16}$

\section{ICAM-1 AND VCAM-1 IN CHILDREN}

ICAM-1 and VCAM-1 expression has been extensively studied in children. Since atherosclerotic lesions seem to have their origin in early childhood and most risk factors for atherosclerosis seem to be influenced by developmental changes mediated by growth and puberty, there is great interest in whether the levels of these particular adhesion molecules fluctuate during childhood and how they are influenced by known risk factors and diseases that predispose subjects to atherosclerosis. Studies so far have failed to demonstrate a definite pathological role for each molecule. Phocas et al. showed a progressive and statistically significant elevation of sICAM-1 during the 1st month of life in 43 healthy neonates. Levels were low on the 1st day of life, reached adult values on the 5th day of life and exceeded adult levels 30 days later. This clear rise is attributable to the bacterial colonization of the neonatal gut and the introduction of feeding. ${ }^{17}$ Malamitsi et al showed that serum levels of VCAM-1 in healthy neonates do not change during early neonatal life unless inflammatory processes or deranged endothelial homeostasis is present. ${ }^{18}$ Stanislas' study, in agreement with two previous studies in healthy children, ${ }^{19,20}$ concluded that sICAM-1 levels are age-related with a decrease between 4 and 17 years old. ${ }^{21}$ Another recently published study, however, demonstrated that children produce higher levels of adhesion molecules. ${ }^{22}$ No difference was found among variable age groups of sVCAM-1 levels. ${ }^{23,24}$ Gender is also known to be associated with cardiovascular risk and both soluble adhesion molecules seem to be sex-related, with boys having higher levels of sICAM-1 and sVCAM-1 compared to girls. ${ }^{22,25}$ This variation of adhesion molecule concentration during childhood has not yet been explained adequately. It is well established that atherosclerotic lesions develop initially during childhood and adolescence in the form of fatty streaks which produce the atherosclerotic lesions of later adult life. ${ }^{4}$ The fact that sICAM- 1 levels are correlated with the development and progression of atherosclerosis, as confirmed in adult studies, and sVCAM-1 levels are high in patients in whom atherosclerotic lesions are already established ${ }^{26,27}$ may partially explain the increased sICAM- 1 concentration in children and the lack of variation of sVCAM-1 levels. Nevertheless, other factors acting through as yet unknown mechanisms may influence the two adhesion molecule variation, complicating the interpretation of study results.

Since the development of atherosclerosis is the result of interaction between genetic backgrounds and environmental influences, it is useful to discuss the biological behavior of children with a familial background of diseases predisposing to atherosclerosis. Levels of sICAM-1 and sVCAM-1 were measured in children from families at high risk for cardiovascular diseases and in children without familial history of cardiovascular disease. sICAM-1 level was significantly higher in the high-risk group in comparison to controls, while there was no significant difference in SVCAM-1. ${ }^{28}$ In addition, healthy offspring of patients with Type 2 diabetes were found to have increased muscular artery stiffness linked to elevated levels of the endothelial activation marker sICAM-1, in comparison with a control group matched for age, adiposity and blood pressure. ${ }^{29}$ Children with heterozygous familiar hypercholesterolemia (FH) exhibit elevated levels of low-density cholesterol from birth onwards and arterial abnormalities consistent with subclinical atherosclerosis as early as within the first decade of life. Stefanadis et al have reported a strong association between increased sICAM-1 levels in $\mathrm{FH}$ children and impaired endothelial function, 
whereas no difference in VCAM-1 expression was found. ${ }^{30}$ In adults results are equivocal: many studies suggest a correlation between SICAM or SVCAM-1 and hypercholesterolemia, while others do not. ${ }^{31-34}$ Equally conflicting are the results regarding triglycerides. ${ }^{35,36}$ Ohta et al. concluded that sVCAM-1 and sICAM-1 in boys are correlated with triglyceride levels and in girls with apoB, apoB to apoA-1 ratio and fractional esterification rate in apolipoprotein B-depleted plasma (FER) high density lipoprotein (HDL). Additionally, sICAM-1 seems to correlate negatively with lipoprotein A-1 levels, HDL particle size in boys and HDL levels in girls.

Consequently, it is suggested that risk factor modification should take place at an earlier age to prevent the chronic elevation of adhesion molecules and thus the risk of future cardiovascular events. ${ }^{20}$ Kazavarakis et al also studied the possible correlation of adhesion molecules with the lipidemic profile in a healthy population of 107 children and confirmed a correlation between sVCAM-1 and triglyceride levels, without this being influenced by sex or lipid fractions. The protective role of HDL was additionally confirmed because it was related to adhesion molecule level reduction. ${ }^{37}$ VCAM-1 correlation with high cholesterol levels has been corroborated in only one study. ${ }^{38}$

A modifiable risk factor such as obesity seems to further enhance the risk. The prevalence of obesity is rapidly increasing in western populations and obesity in childhood is established as a predictive factor for adult cardiovascular disease, associated with endothelial dysfunction and arterial wall thickening. ${ }^{39}$ Recent studies provide evidence that overweight and obese children present with increased circulating levels of sICAM-1 and VCAM-1,,$^{22,40-43}$ while only one study suggests that there is no difference between obese and normal weight children. ${ }^{44}$ Overweight children have elevated levels of circulating soluble adhesion molecules closely related to excess body fat ${ }^{45}$ body mass index (BMI) and insulin resistance (IR). ${ }^{22} \mathrm{It}$ should be noted that IR is a well-established consequence of excess weight and obesity ${ }^{46,47}$ Lee et al showed that in Caucasian, but not in African, youths, (8-19 years) IR was significantly associated with the biomarkers of endothelial dysfunction, ICAM-1 and E-selectin. ${ }^{45,48}$ This study also suggested that racial differences in circulating adhesion molecules are already present during childhood. Although uncertain, it is plausible that higher visceral adipose tissue in Caucasians compared to African Americans may in part explain this racial differential in endothelial biomarkers. ${ }^{49}$ In recent studies, obese children displayed significantly elevated sICAM-1, IR ${ }^{50}$ and C-reactive protein (CRP) values. ${ }^{22,43,51}$ A correlation between the adhesion molecules ${ }^{52}$ and 8-iso-PGF2 factor (index of oxidative stress, endothelial and platelet activation) was also observed, revealing that early endothelial and platelet activation occurs in obese children. ${ }^{43}$ This elevation of adhesion molecule levels may not be associated with atherosclerotic lesion formation. Indeed, in a study of 104 children where intima medial thickness (IMT) was used as a measure of atherosclerotic lesion extent, no significant correlation was revealed between adhesion molecules and IMT. SICAM-1 levels increased in parallel with the degree of the severity of the lesions but in a non-statistically significant manner. ${ }^{53}$ Higher levels of both molecules were evident in obese children with concomitant hypertension, while in normal weight but hypertensive children only the sICAM-1 levels were higher than normal. There was also a correlation of total cholesterol levels with sVCAM-1 levels. ${ }^{38}$ In another study of 31 hypertensive and 9 healthy, normotensive children and young adults, hypertensive children displayed significantly higher levels of VCAM-1. ${ }^{54}$ In adults, however, hypertension is not correlated with sVCAM-1 and sICAM-1. . $^{32,35}$ Interestingly, changes in body mass index are associated with changes in inflammatory and endothelial biomarkers. ${ }^{50} \mathrm{BMI}-$ SDS reduction is correlated with changes in SICAM-1 levels both in children and adults. ${ }^{55}$ In adults, BMI and obesity are correlated with sICAM-1 levels, while at the same time BMI and obesity reduction result in lowering both ICAM-1 and VCAM- $1 .{ }^{56}$ Weight loss in all these cases seems to improve the status of atherosclerosis risk factors while leading simultaneously to the lowering of adhesion molecules concentration. ${ }^{56}$

Diabetes mellitus (DM) has been recognized as an important risk factor for cardiovascular disease in adults. ${ }^{57}$ It has also been suggested that it is a risk factor for atherosclerotic lesion in the coronary vessels of children aged 2-15 years old suffering from diabetes mellitus type 1 (T1DM) ${ }^{58}$ Hyperglycemia per se as well as increased production of free radi- 
cals (markers of oxidative stress) are crucial for the increased expression of adhesion molecules in young patients with T1DM..$^{59}$ Therefore, the role of diabetes mellitus in combination with adhesion molecules has been studied. Abnormally high levels of sICAM-1 and SVCAM-1 are reported in children with T1DM compared to controls. A significant correlation also exists between $\mathrm{HbA} 1 \mathrm{c}$ and sICAM-1 in diabetics compared to controls. ${ }^{59}$ Triolo et al showed not only that patients with T1DM had increased levels of sICAM-1 compared to healthy subjects but, moreover, that levels of sICAM-1 were significantly higher in patients with positive IgG anti-endothelial cell antibodies. ${ }^{60}$ Furthermore, healthy Finnish schoolchildren with positive IA-2A autoantibodies have increased levels of sICAM-1 compared to seronegative subjects. Since IA-2A autoantibodies are the last autoantibodies to appear in the prediabetic process, data suggest that increased levels of SICAM-1 are a characteristic feature of late preclinical T1DM. ${ }^{24}$ The same group has reported high levels of circulating sICAM-1 and L-selectin in the siblings of affected children who have the highest risk of progression to clinical T1DM. ${ }^{61}$ In adults, a correlation of diabetes mellitus with increased levels of sICAM-1 has also been demonstrated. ${ }^{62}$

In conclusion, sICAM-1 appears to reflect the extension of atherosclerotic lesions and may be a predictive factor for future cardiovascular events in adults, ${ }^{31,62,63}$ while sVCAM-1 mainly reflects the extension of atherosclerosis and appears to be a prognostic factor for patients with extensive and already established atherosclerotic disease..$^{64,65}$ The presence of atherosclerosis risk factors in childhood combined with increased levels of adhesion molecules suggest that atherosclerotic vascular lesions develop and progress during the first years of life. Early recognition and intervention is therefore crucial, especially in high-risk group children who are prone to developing cardiovascular disease in the future. The measurement of these two adhesion molecules may prove a useful tool to prevent atherosclerosis and cardiovascular consequences in adult life, although determination of their precise role in atherosclerosis and the importance of their serum levels in childhood is as yet problematical. Since, based on current studies, results remain ambiguous and sometimes conflicting, more studies must be conducted in order to determine the role of these molecules.

\section{REFERENCES}

1. Barker DJ, 1995 Fetal origins of coronary heart disease. BMJ 311:171-174.

2. Seidman DS, Laor A, Gale R, Stevenson DK, Danon YL, 1991 A longitudinal study of birth weight and being overweight in late adolescence. Am J Dis Child 145: 782-785.

3. Eriksson JG, Forsen T, Tuomilehto J, Osmond C, Barker DJ, 2001 Early growth and coronary heart disease in later life: longitudinal study. Br Med J 322: 949-953.

4. Libby P, 2000 Changing concepts of atherogenesis. J Intern Med 247: 349-358.

5. Price DT, Loscalzo J, 1999 Cellular adhesion molecules and atherogenesis. Am J Med, 107: 85-97.

6. Ramos KS, Partridge CR, Teneng I, 2007 Genetic and molecular mechanisms of chemical atherogenesis. Mutat Res 62: 18-30.

7. van de Stolpe A, van der Saag PT, 1996 Intercellular adhesion molecule-1. J Mol Med (Berlin, Germany) 74: 13-33.

8. Hubbard AK, Rothlein R, 2000 Intercellular adhesion molecule-1 (ICAM-1) expression and cell signaling cascades. Free Radic Biol Med 28:1379-1386.

9. van Buul JD, Kanters E, Hordijk PL, 2007 Endothelial signaling by Ig-like cell adhesion molecules. Arterioscler Thromb Vasc Biol 27: 1870-1876.

10. Witkowska AM, Borawska MH, 2004 Soluble intercellular adhesion molecule-1 (sICAM-1): an overview. Eur Cytokine Netw 15: 91-98.

11. Elangbam CS, Qualls CW, Jr., Dahlgren RR, 1997 Cell adhesion molecules-update. Vet Pathol 34: 61-73.

12. Mazo IB, Gutierrez-Ramos JC, Frenette PS, Hynes RO, Wagner DD, von Andrian UH, 1998 Hematopoietic progenitor cell rolling in bone marrow microvessels: parallel contributions by endothelial selectins and vascular cell adhesion molecule 1. J Exp Med 188: 465-474.

13. Carter RA, Wicks IP, 2001 Vascular cell adhesion molecule 1 (CD106): a multifaceted regulator of joint inflammation. Arthritis Rheum 44: 985-994.

14. Kelly KA, Allport JR, Yu AM, et al, 2007 SPARC is a VCAM-1 counter-ligand that mediates leukocyte transmigration. J Leukoc Biol 81: 748-756.

15. Postigo AA, Teixido J, Sanchez-Madrid F, 1993 The alpha 4 beta 1/VCAM-1 adhesion pathway in physiology and disease. Res Immunol 144: 723-735; discussion 754-762.

16. Leca G, Mansur SE, Bensussan A, 1995 Expression of VCAM-1 (CD106) by a subset of TCR gamma delta-bearing lymphocyte clones. Involvement of a metalloprotease in the specific hydrolytic release of the soluble isoform. J Immunol 154: 1069-1077.

17. Phocas I, Sarandakou A, Giannaki G, Malamitsi-Puchner 
A, Rizos D, Zourlas PA, 1998 Soluble intercellular adhesion molecule-1 in newborn infants. Eur J Pediatr 157: $153-156$

18. Malamitsi-Puchner A, Tziotis J, Mastorakos G, Protonotariou E, Creatsas G, 2000 Adhesion molecules in early neonatal life. Biol Neonate 78: 65-67.

19. Nash MC, Wade AM, Shah V, Dillon MJ, 1996 Normal levels of soluble E-selectin, soluble intercellular adhesion molecule-1 (sICAM-1), and soluble vascular cell adhesion molecule-1 (sVCAM-1) decrease with age. Clin Exp Immunol 103: 167-170.

20. Ohta T, Saku K, Takata K, Adachi N, 1999 Soluble vascular cell-adhesion molecule-1 and soluble intercellular adhesion molecule-1 correlate with lipid and apolipoprotein risk factors for coronary artery disease in children. Eur J Pediatr 158: 592-598.

21. Ponthieux A, Herbeth B, Droesch S, Haddy N, Lambert D, Visvikis S, 2004 Biological determinants of serum ICAM-1, E-selectin, P-selectin and L-selectin levels in healthy subjects: the Stanislas study. Atherosclerosis 172: 299-308.

22. Al-Isa AN, Thalib L, Akanji AO, 2010 Circulating markers of inflammation and endothelial dysfunction in Arab adolescent subjects: reference ranges and associations with age, gender, body mass and insulin sensitivity. Atherosclerosis 208: 543-549.

23. Sack U, Burkhardt U, Borte M, Schadlich H, Berg K, Emmrich F, 1998 Age-dependent levels of select immunological mediators in sera of healthy children. Clin Diagn Lab Immunol 5: 28-32.

24. Toivonen A, Kulmala P, Rahko J, Ilonen J, Knip M, 2004 Soluble adhesion molecules in Finnish schoolchildren with signs of preclinical type 1 diabetes. Diabetes Metab Res Rev 20: 48-54.

25. Chen Y, Osika W, Dangardt F, Gan LM, Strandvik B, Friberg P, High levels of soluble intercellular adhesion molecule-1, insulin resistance and saturated fatty acids are associated with endothelial dysfunction in healthy adolescents. Atherosclerosis 211: 638-642.

26. Tzoulaki I, Murray GD, Lee AJ, Rumley A, Lowe GD, Fowkes FG, 2005 C-reactive protein, interleukin-6, and soluble adhesion molecules as predictors of progressive peripheral atherosclerosis in the general population: Edinburgh Artery Study. Circulation 112:976-983.

27. Pradhan AD, Rifai N, Ridker PM, 2002 Soluble intercellular adhesion molecule-1, soluble vascular adhesion molecule-1, and the development of symptomatic peripheral arterial disease in men. Circulation 106: 820-825.

28. Wojakowski W, Gminski J, 2001 Soluble ICAM-1, VCAM-1 and E-selectin in children from families with high risk of atherosclerosis. Int J Mol Med 7: 181-185.

29. McEleavy OD, McCallum RW, Petrie JR, et al, 2004 Higher carotid-radial pulse wave velocity in healthy offspring of patients with Type 2 diabetes. Diabet Med 21: $262-266$.
30. Charakida M, Tousoulis D, Skoumas I, et al, 2009 Inflammatory and thrombotic processes are associated with vascular dysfunction in children with familial hypercholesterolemia. Atherosclerosis 204: 532-537.

31. Ridker PM, Hennekens CH, Roitman-Johnson B, Stampfer MJ, Allen J, 1998 Plasma concentration of soluble intercellular adhesion molecule 1 and risks of future myocardial infarction in apparently healthy men. Lancet 351: 88-92.

32. Hwang SJ, Ballantyne CM, Sharrett AR, et al, 1997 Circulating adhesion molecules VCAM-1, ICAM-1, and E-selectin in carotid atherosclerosis and incident coronary heart disease cases: the Atherosclerosis Risk In Communities (ARIC) study. Circulation 96: 42194225 .

33. de Lemos JA, Hennekens CH, Ridker PM, 2000 Plasma concentration of soluble vascular cell adhesion molecule-1 and subsequent cardiovascular risk. J Am Coll Cardiol 36: 423-426.

34. Zhu Y, Liao HL, Lin JH, Verna L, Stemerman MB, 1999 Low-density lipoprotein augments interleukin1 -induced vascular adhesion molecule expression in human endothelial cells. Atherosclerosis 144:357-365.

35. Malik I, Danesh J, Whincup P, et al, 2001 Soluble adhesion molecules and prediction of coronary heart disease: a prospective study and meta-analysis. Lancet 358: 971-976.

36. Benitez MB, Cuniberti L, Fornari MC, et al, 2008 Endothelial and leukocyte adhesion molecules in primary hypertriglyceridemia. Atherosclerosis 197: 679-687.

37. Kavazarakis E, Moustaki M, Gourgiotis D, et al, 2002 The impact of serum lipid levels on circulating soluble adhesion molecules in childhood. Pediatr Res 52: 454458.

38. Glowinska B, Urban M, Peczynska J, Florys B, 2005 Soluble adhesion molecules (sICAM-1, sVCAM-1) and selectins ( $\mathrm{sE}$ selectin, sP selectin, sL selectin) levels in children and adolescents with obesity, hypertension, and diabetes. Metabolism 54: 1020-1026.

39. Tounian P, Aggoun Y, Dubern B, et al, 2001 Presence of increased stiffness of the common carotid artery and endothelial dysfunction in severely obese children: a prospective study. Lancet 358: 1400-1404.

40. Desideri G, De Simone M, Iughetti L, et al, 2005 Early activation of vascular endothelial cells and platelets in obese children. J Clin Endocrinol Metab 90: 3145-3152.

41. Suheyl Ezgu F, Hasanoglu A, Tumer L, Ozbay F, Aybay C, Gunduz M, 2005 Endothelial activation and inflammation in prepubertal obese Turkish children. Metabolism 54: 1384-1389.

42. Caballero AE, Bousquet-Santos K, Robles-Osorio L, et al, 2008 Overweight Latino children and adolescents have marked endothelial dysfunction and subclinical vascular inflammation in association with excess body fat and insulin resistance. Diabetes Care 31: 576-582.

43. Fontana L, Giagulli C, Minuz P, Lechi A, Laudanna C, 
2001 8-Iso-PGF2 alpha induces beta 2-integrin-mediated rapid adhesion of human polymorphonuclear neutrophils: a link between oxidative stress and ischemia/reperfusion injury. Arterioscler Thromb Vasc Biol 21: 55-60.

44. Kapiotis S, Holzer G, Schaller G, et al, 2006 A proinflammatory state is detectable in obese children and is accompanied by functional and morphological vascular changes. Arterioscler Thromb Vasc Biol 26: 2541-2546.

45. Lee S, Gungor N, Bacha F, Arslanian S, 2007 Insulin resistance: link to the components of the metabolic syndrome and biomarkers of endothelial dysfunction in youth. Diabetes Care 30: 2091-2097.

46. Sinha R, Fisch G, Teague B, et al, 2002 Prevalence of impaired glucose tolerance among children and adolescents with marked obesity. N Engl J Med 346: 802-810.

47. Barja S, Arteaga A, Acosta AM, Hodgson MI, 2003 Insulin resistance and other expressions of metabolic syndrome in obese Chilean children. Rev Med Chil, 131: 259-268.

48. Lee S, Bacha F, Gungor N, Arslanian S, 2008 Comparison of different definitions of pediatric metabolic syndrome: relation to abdominal adiposity, insulin resistance, adiponectin, and inflammatory biomarkers. J Pediatr 152: 177-184.

49. Hill JO, Sidney S, Lewis CE, Tolan K, Scherzinger AL, Stamm ER, 1999 Racial differences in amounts of visceral adipose tissue in young adults: the CARDIA (Coronary Artery Risk Development in Young Adults) study. Am J Clin Nutr 69: 381-387.

50. Martos R, Valle M, Morales RM, Canete R, Gascon F, Urbano MM, 2009 Changes in body mass index are associated with changes in inflammatory and endothelial dysfunction biomarkers in obese prepubertal children after 9 months of body mass index SD score loss. Metabolism 58: 1153-1160.

51. Valle Jimenez M, Estepa RM, Camacho RM, Estrada RC, Luna FG, Guitarte FB, 2007 Endothelial dysfunction is related to insulin resistance and inflammatory biomarker levels in obese prepubertal children. Eur J Endocrinol 156: 497-502.

52. Garanty-Bogacka B, Syrenicz M, Syrenicz A, Gebala A, Walczak M, 2005 Relation of acute-phase reaction and endothelial activation to insulin resistance and adiposity in obese children and adolescents. Neuro Endocrinol Lett 26:473-479.

53. Beauloye V, Zech F, Tran HT, Clapuyt P, Maes M, Brichard SM, 2007 Determinants of early atherosclerosis in obese children and adolescents. J Clin Endocrinol Metab 92: 3025-3032.

54. Goonasekera CD, Shah V, Rees DD, Dillon MJ, 2000
Vascular endothelial cell activation associated with increased plasma asymmetric dimethyl arginine in children and young adults with hypertension: a basis for atheroma? Blood Press 9: 16-21.

55. Hamdy O, Ledbury S, Mullooly C, et al, 2003 Lifestyle modification improves endothelial function in obese subjects with the insulin resistance syndrome. Diabetes Care 26: 2119-2125.

56. Hope SA, Meredith IT, 2003 Cellular adhesion molecules and cardiovascular disease. Part II. Their association with conventional and emerging risk factors, acute coronary events and cardiovascular risk prediction. Intern Med J 33: 450-462.

57. Berry JD, Dyer A, Cai X, et al, 2012 Lifetime risks of cardiovascular disease. N Engl J Med 366:321-329.

58. Libby P, Nathan DM, Abraham K, et al, 2005 Report of the National Heart, Lung, and Blood Institute-National Institute of Diabetes and Digestive and Kidney Diseases Working Group on Cardiovascular Complications of Type 1 Diabetes Mellitus. Circulation 111: 3489-3493.

59. Seckin D, Ilhan N, Ilhan N, Ertugrul S, 2006 Glycaemic control, markers of endothelial cell activation and oxidative stress in children with type 1 diabetes mellitus. Diabetes Res Clin Pract 73: 191-197.

60. Triolo G, Accardo-Palumbo A, Carbone MC, Ferrante A, Casiglia D, Giardina E, 1998 IgG anti-endothelial cell antibodies (AECA) in type I diabetes mellitus; induction of adhesion molecule expression in cultured endothelial cells. Clin Exp Immunol 111: 491-496.

61. Toivonen A, Kulmala P, Savola K, Akerblom HK, Knip M, 2001, The Childhood Diabetes In F: Soluble adhesion molecules in preclinical type 1 diabetes. The Childhood Diabetes in Finland Study Group. Pediatr Res 49: 24-29.

62. Keaney JF Jr, Massaro JM, Larson MG, et al, 2004 Heritability and correlates of intercellular adhesion molecule-1 in the Framingham Offspring Study. J Am Coll Cardiol 44: 168-173.

63. Luc G, Arveiler D, Evans A, et al, 2003, Circulating soluble adhesion molecules ICAM-1 and VCAM-1 and incident coronary heart disease: the PRIME Study. Atherosclerosis 170: 169-176.

64. Rohde LE, Lee RT, Rivero J, et al, 1998 Circulating cell adhesion molecules are correlated with ultrasoundbased assessment of carotid atherosclerosis. Arterioscler Thromb Vasc Biol 18: 1765-1770.

65. De Caterina R, Basta G, Lazzerini G, et al, 1997 Soluble vascular cell adhesion molecule-1 as a biohumoral correlate of atherosclerosis. Arterioscler Thromb Vasc Biol 17: 2646-2654. 